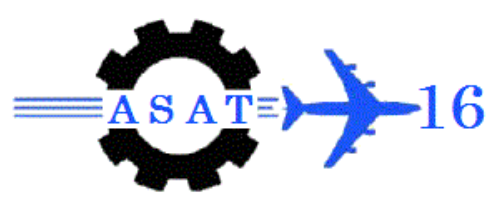

\title{
Aluminum oxide doped/Aniline hydrochloride films for gamma radiation detection
}

\author{
Ahmed Omar*a ${ }^{* a}$ Ahmad Baraka*, Ahmed H.Zaki* ${ }^{\mathrm{b}}$ and Karam A.Sharshar** \\ ah_omar_mtc@hotmail.com, ${ }^{\mathrm{b}}$ ahafiz@lycos.com

\begin{abstract}
In the present work, aniline doped aluminum oxide films were prepared by the Sol-Gel technique for gamma radiation dosimetry. Aniline (AN) was suggested to enhance the electrical conductivity of films. Measured conductivity due to gamma radiation was studied in the $0-10 \mathrm{kGy}$ dose range. Conductivity was enhanced with AN doping. Conductivity and sensitivity values up to $150\left(\mu \Omega^{-1} \cdot \mathrm{cm}^{-1}\right)$ and $3.75\left(\mathrm{~mA} \cdot \mathrm{cm}^{-2} \cdot \mathrm{kGy}^{-1}\right)$ respectively were achieved at $0.25 \mathrm{~g}$ AN content and $25 \mathrm{~V}$ bias.
\end{abstract}

Keywords: Aniline doped aluminum oxide films, Gamma radiation and Gamma Dosimetry.

\section{Introduction}

Radiation dosimeters are utilized in a wide range of applications; medicine, industry, and science. Exposure to ionizing radiation (X-and $\gamma$-ray are sounding examples) may happen in these applications and adequate detection of radiation is essential for environmental safety. A radiation dosimeter is a system (basically material) that either directly or indirectly quantitatively determines the absorbed dose of ionizing radiation. The dosimeter has to possess a physical or a chemical property that linearly changes with radiation dose in a proper manner to be calibrated.

In order to be useful, radiation dosimeters should exhibit several desirable characteristics; reasonable sensitivity, linear performance over the intended energy range and acceptable reliability. The choice of a particular dosimeter type for an application depends on the X- or $\gamma$ ray energy range. So it is important for environmental safety to produce different systems for ionizing radiation detection.

Metal oxides are famous type of materials being used for dosimetry. It is utilized in the film form. Most of the produced films are prepared by different techniques of deposition such as thermal evaporation and screen printing. The main property that changes with radiation is the electrical conductivity. Khalil Arshak et al studied the properties of the materials that undergone changes by the influence of gamma-rays. The degree of these changes could serve as a measure of the received radiation dose [1].

J. Corcoran et al interested in investigation of the feasibility of the use of titanium dioxide $\left(\mathrm{TiO}_{2}\right)$, zinc oxide $(\mathrm{ZnO})$, copper oxide $(\mathrm{CuO})$ and Cadmium oxide $(\mathrm{CdO})$ in the development of thick film gamma radiation sensors with instantaneous dosage readouts [2].

\footnotetext{
* Military Technical College

*** Egyptian Atomic Energy Authority
} 
Zinc oxide $(\mathrm{ZnO})$ and stannous oxide $\left(\mathrm{SnO}_{2}\right)$ thick film capacitors with inter-digitated electrodes were used as gamma radiation sensors. Irradiation with doses from $1 \mathrm{mGy}$ to 2.3 mGy caused the increase in the values of $\mathrm{ZnO}$ capacitor, whereas counterpart $\mathrm{SnO}_{2}$ sensors were more sensitive to lower doses of radiation in the range of 0-0.46 $\mathrm{mGy}$ [3].

Thick films of nickel oxide $(\mathrm{NiO})$ in the form of pn-junction and planar structure were investigated for gamma radiation dosimetry purposes. They showed an increase in the value of current with increase in radiation dose up to certain level [4].

The current-voltage $(I-V)$ characteristics of metal oxides films, such as nickel oxide $(\mathrm{NiO})$, cerium oxide $\left(\mathrm{CeO}_{2}\right)$ and indium oxide $\left(\mathrm{In}_{2} \mathrm{O}_{3}\right)$ were measured at different doses. Results show that the current is increased with the increase in radiation dose to a certain level, which indicated the working dose region of the device [5].

Tellurium dioxide $\left(\mathrm{TeO}_{2}\right)$, indium oxide $\left(\mathrm{In}_{2} \mathrm{O}_{3}\right)$, silicon monoxide $(\mathrm{SiO})$, nickel oxide $(\mathrm{NiO})$ and their mixtures in different proportions in the forms of thin and thick films were used as radiation sensing layers. Detection of radiation was performed based on the fact that both electrical and optical properties of the materials undergo changes upon the exposure to gamma radiation. Throughout this work oxides are regarded as an appropriate cost-effective material for radiation sensing [6].

The current-voltage $(I-V)$ characteristics of the $\mathrm{TeO}_{2}$ thin films of different thicknesses, prepared by thermal evaporation in vacuum, have been carried out for different ranges of gamma radiation doses. This study show that the current density increases almost linearly with the gamma radiation dose and the $\mathrm{TeO}_{2}$ thin films of thicknesses in the range 300-1500 $\mathrm{nm}$ are expected to possess sensitivities in the range $1.2-37.0 \mathrm{nA} / \mathrm{cm}^{2} / \mathrm{mGy}$ and detection limits in the range $0.22-2.16 \mathrm{mGy}$ [7].

The effect of gamma radiation on aluminum oxide $\mathrm{Al}_{2} \mathrm{O}_{3}$ dielectric in MOS structure was studied, the result was compared with conventional MOS capacitors with a $\mathrm{SiO}_{2}$ dielectric. The effect of radiation has been determined from the valance band shift in $(C-V)$ curves. The amount of charge induced by the radiation has been calculated and compared with the response of MOS capacitors with $\mathrm{SiO}_{2}$ with the same and different thicknesses [8].

In some cases, conductivity of metal oxide film is enhanced by doping or adding a certain percentage of a conductive polymer. Nano-crystalline $\mathrm{TiO}_{2}$ films were prepared using sol-gel spin coating method to deposit a film on surface of polyaniline between two substrates of conductive glass ITO, $(I-V)$ characteristic for this structure indicated that a radiation sensitive pn-junction at the interface between $\mathrm{TiO}_{2}$ and polyaniline was formed [9].

Another type of dosimeters is based on applying conductive polymers. The conductivity of the famous conductive polymer is modulated by radiation. It is prepared in the form of non conducting polymer and blended with different polymer to cast a film which upon irradiation is transferred to conductive polyaniline.

In a typical work, polyaniline was blended with chlorine-containing polymers, copolymers and HCl-releasing compounds to determine their radiation response in terms of induced conductivities. The results showed that ionizing radiation induced controllable conductivity in these blends [10]. Polyaniline pellets were investigated as radiation sensor. The pellets were formed by hydraulic press. The electrical conductivity before and during radiation exposure (using alpha, beta, gamma radiation sources) was measured and evaluated [11].

Polyaniline/polyacrylic acid (PAA) thin film composites were prepared. The four-point conductivity measurements showed that composite exhibits a linear response for the dose range from 0 to $5 \mathrm{kGy}$ [12]. The effect of ionizing radiation such as gamma radiation and neutron have been studied on conducting polyaniline for various doses and spectroscopic methods such as UV-VIS spectroscopy and I-V measurements have been used to characterize the effect of gamma radiation on polyaniline conducting polymer[13]. 
PANI films were prepared, having average thickness of $2 \mu \mathrm{m}$. These samples were exposed to varying gamma radiation dose up to $10 \mathrm{kGy}$. It was found the conductivity has a regular change with dose that could be represented linearly. This increment in the conductivity of the PANI film shows the effect of gamma radiation dose on the film due to the transformation from non conductive polyaniline form to conductive polyaniline form, which can be used as a sensitive detector of gamma radiation in that range [14].

In this work aluminum oxide film was deposited by using the simple sol-gel technique which is considered as new method to be used in this field of fabrication of metal oxide semiconductor sensor for gamma radiation in which evaporation and screen printing techniques are the most common. Also the effect of gamma radiation on aluminum oxide film was studied through the determination of $(I-V)$ characteristic using a simple electronic circuit.

Polyaniline-doped aluminum oxide films were prepared by sol gel technique to ensure the homogenous presence of post-irradiation conductive polyaniline throughout the aluminum oxide structure. This is highly suggesting the noticeable enhancement of conductivity of the produced films to make a combination between inorganic and organic material to be used for gamma rays detection.

\section{Experimental.}

\subsection{Materials.}

Aluminum nitrate nona-hydrate $\left(\mathrm{Al}\left(\mathrm{NO}_{3}\right)_{3} .9 \mathrm{H}_{2} \mathrm{O}, \mathrm{MWT}=374.98\right.$, Fine grad $)$, absolute ethanol $\left(\mathrm{C}_{2} \mathrm{H}_{6} \mathrm{O}, \mathrm{MWT}=46.07\right)$, Propylene oxide $\left(\mathrm{C}_{3} \mathrm{H}_{6} \mathrm{O}, \mathrm{MWT}=58.08\right.$, Aldrich $)$, Distilled water and Aniline hydrochloride as a monomer (AN, $\mathrm{Mw}=129.59 \mathrm{~g} / \mathrm{mol}$, Aldrich).

\subsection{Preparation of aluminum oxide sol solution doped with Aniline hydrochloride.}

Aluminum nitrate was dissolved in absolute ethanol and propylene oxide was added to the solution. Also, AN $(0.10 \mathrm{~g}, 0.15 \mathrm{~g}, 0.20 \mathrm{~g}$ and $0.25 \mathrm{~g})$ was added to solution. Final sol gel solution was spread over glass substrate using spin coating technique.

\subsection{Preparation of thin film using spin coating technique.}

Five solutions of Aluminum oxide sol doped with different AN content were prepared based on the blank sample, the contents of $\mathrm{AN}$ were $(0.10 \mathrm{~g}, 0.15 \mathrm{~g}, 0.20 \mathrm{~g}$ and $0.25 \mathrm{~g})$, each solution was used for preparing five sample of films on glass substrate in the form of resistor. The effective part of the films has the dimensions of $2 \mathrm{~cm}$ length, $1 \mathrm{~mm}$ width and $2 \mu \mathrm{m}$ thickness. Contacts were aluminum layer stacked on glass substrate as electrical connector as shown in figure 1.

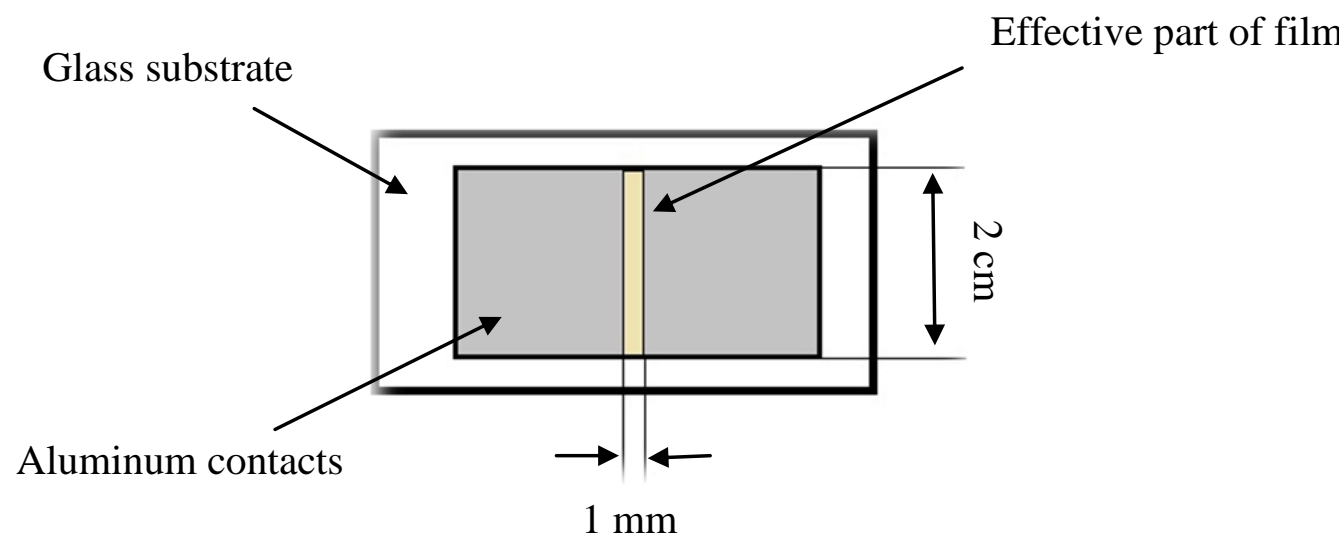

Fig. 1.Coplanar structure of a film for the current-voltage measurements 
After complete formation of designed films and drying in air for one hour, the films were dried in oven at $50{ }^{\circ} \mathrm{C}$ for two hours then temperature is increased to $75{ }^{\circ} \mathrm{C}$ for half an hour to avoid formation of cracks in the films.

\subsection{Gamma irradiation.}

The films were exposed to gamma radiation using ${ }^{60} \mathrm{Co}$ radiation facility (Gamma Chamber 5000) at a constant dose rate $(26.66 \mathrm{~Gy} / \mathrm{min})$ at room temperature. The samples were irradiated by a linearly increased dose with $2 \mathrm{kGy}$ step up to $10 \mathrm{kGy}$.

\subsection{Electrical measurements for irradiated films.}

The conductivity of the prepared films was determined by measuring the induced current by applying different voltages in the range $0-25 \mathrm{~V}$. In the present work, the current induced in the samples due to voltage applied by (DC power supply) voltage source was measured. For current measurement, samples of planer structure irradiated with different doses up to10 kGy were attached to a simple electrical measurement circuit as resistance type. For each film there were five samples which were prepared under same conditions and electrical measurements were averaged to reduce the error.

\section{Result and Analysis.}

\subsection{Current - Voltage analysis for AN free Aluminum oxide film}

The typical current-voltage (I-V) characteristics for the coplanar structure of the AN free aluminium oxide film, exposed to different levels of the gamma radiation dose, are shown in figure 2 and linear fitting equations are shown in Table 1.

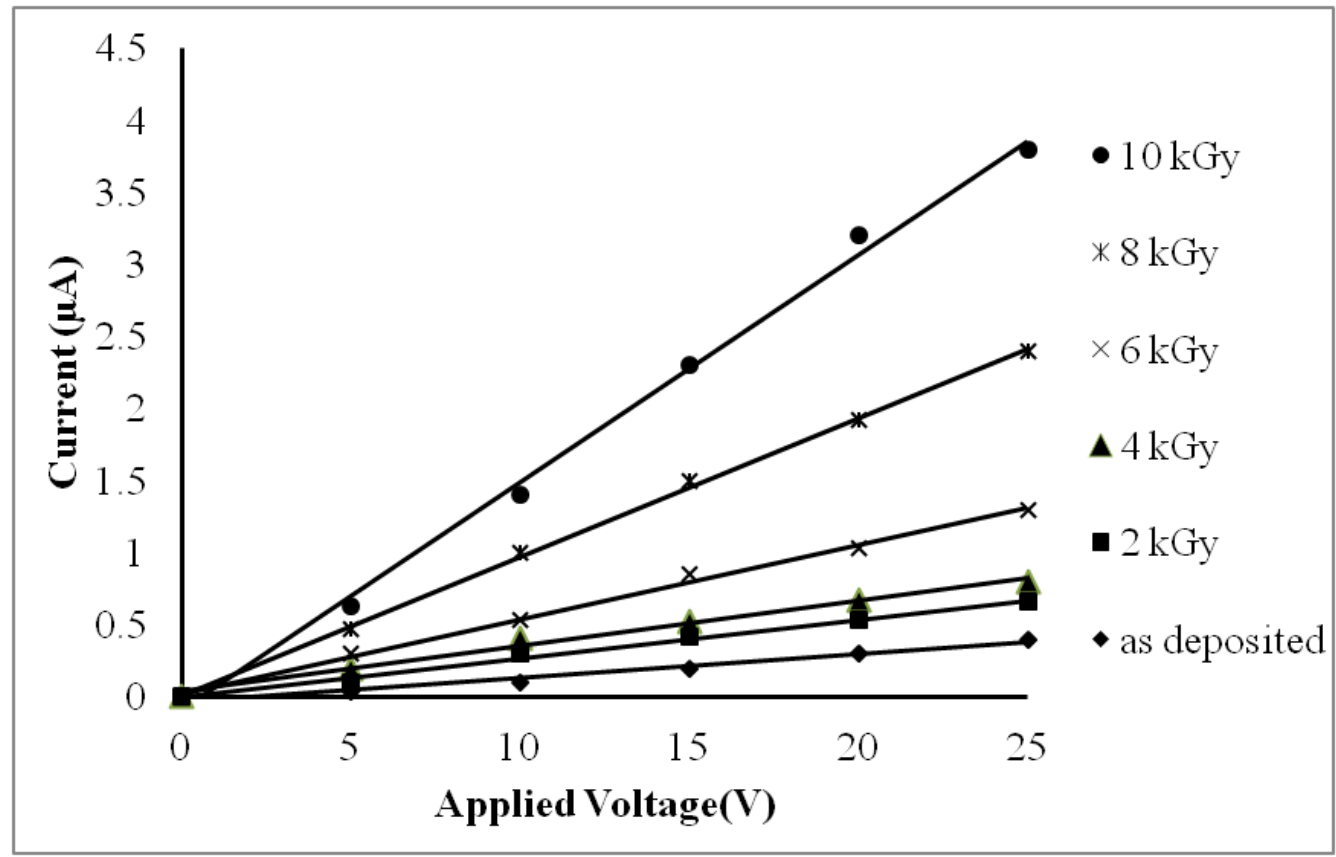

Fig. 2. Typical current-voltage characteristics for Aluminium oxide film free from AN exposed to different levels of the gamma radiation dose. 
Table 1. The fitted linear equations represent the relation between induced current and applied volt for Aluminium oxide film free from AN

\begin{tabular}{ccc}
\hline $\begin{array}{c}\text { Dose } \\
\mathrm{kGy}\end{array}$ & $\begin{array}{c}\text { Fitted linear } \\
\text { equations }\end{array}$ & $\begin{array}{c}\text { Correlation factor } \\
\left(\mathrm{R}^{2}\right)\end{array}$ \\
\hline 0 & $I=0.0165 V-0.0324$ & 0.976 \\
2 & $I=0.0269 V-0.0014$ & 0.991 \\
4 & $I=0.0315 V+0.0390$ & 0.987 \\
6 & $I=0.0515 V+0.0248$ & 0.996 \\
8 & $I=0.0963 V+0.0114$ & 0.999 \\
10 & $I=0.1578 V-0.0838$ & 0.9962 \\
\hline \multicolumn{3}{c}{$I$ is the induced current $(\mu \mathrm{A})$ and $V$ is applied volt $(V)$}
\end{tabular}

Figure 2 indicates that the induced current due to gamma irradiation increases in level as gamma radiation dose increases up to $10 \mathrm{kGy}$ with applied voltage from 0 to $25 \mathrm{~V}$. Subsequently, these data were analyzed to obtain changes of the current density with the gamma radiation dose at different voltages applied to the coplanar structure of the thin films as shown in figure 3 where it showed direct proportion between the current density and gamma radiation dose.

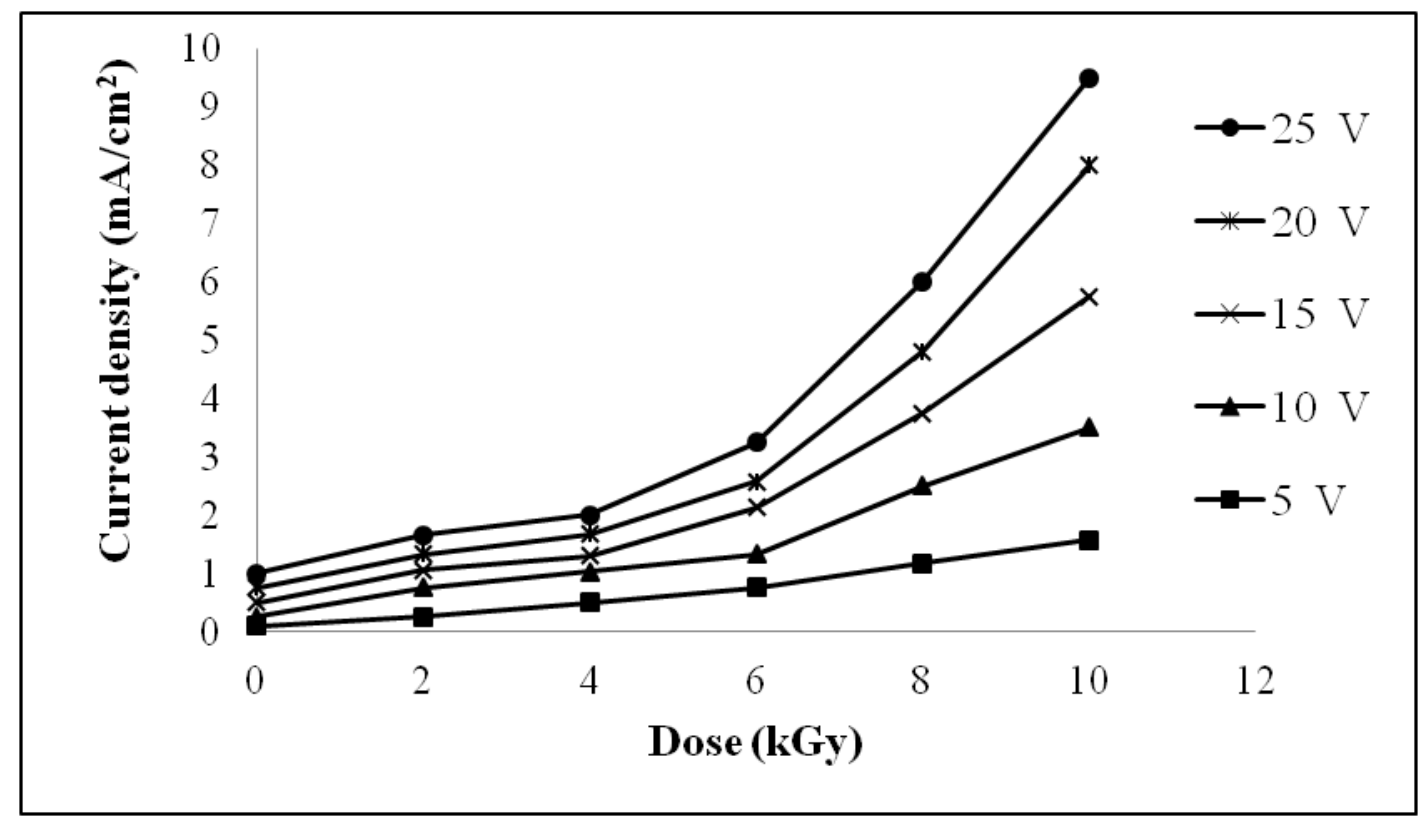

Fig. 3. Dependence of the current density with gamma radiation dose for $\mathrm{AN}$-free aluminium oxide film

From the linear part of each of the current density versus gamma radiation dose plots, the sensitivity was estimated. The sensitivity represents the change in the current density per unit change of the gamma radiation dose. Sensitivities were found to be in the range 0.16-0.98 $\mathrm{mA} / \mathrm{cm}^{2} / \mathrm{kGy}$ as shown in Table 2 which means that sensitivity of the film increase as the applied voltage increase. 
Table 2. Sensitivities (in $\mathrm{mA} / \mathrm{cm}^{2} / \mathrm{kGy}$ ) at different voltages applied to coplanar structure film free from AN.

\begin{tabular}{cc}
\hline $\begin{array}{c}\text { Applied voltage } \\
(\mathrm{V})\end{array}$ & $\begin{array}{c}\text { Sensitivity } \\
\left(\mathrm{mA} / \mathrm{cm}^{2} / \mathrm{kGy}\right)\end{array}$ \\
\hline 5 & 0.165625 \\
10 & 0.34375 \\
15 & 0.5875 \\
20 & 0.834375 \\
25 & 0.98125 \\
\hline
\end{tabular}

Figure 4 shows the measured conductivity changes against different levels of gamma radiation dose which indicates conductivity increase versus gamma radiation dose.

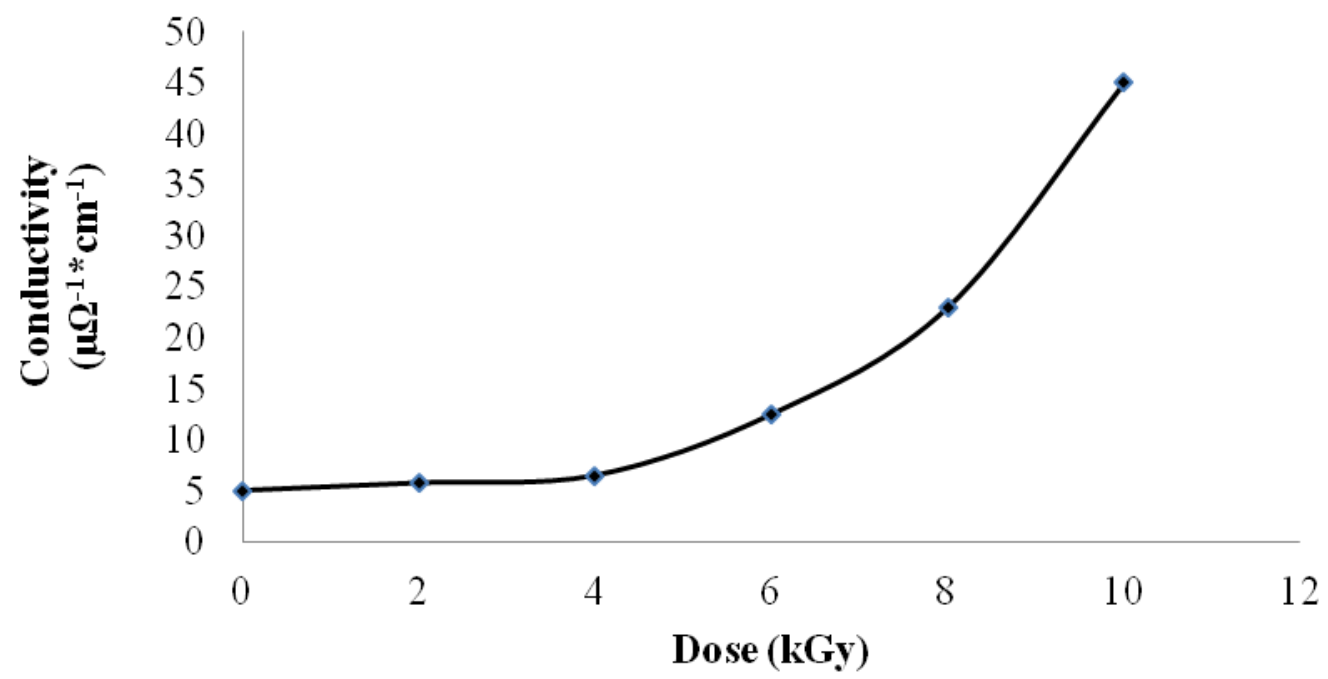

Fig. 4. Dependence of conductivity with gamma radiation dose for AN-free aluminium oxide film

3.2 Current \& Voltage analysis for Aluminum oxide film doped with (0.1g, $0.15 g$, $0.2 \mathrm{~g}$ and $0.25 \mathrm{gAN}$ )

Figures 5, 6, 7 and 8 show the Current-Volt (I-V) chractrastiec for coplanar structure of Aluminum oxide film doped samples $(0.1 \mathrm{~g}, 0.15 \mathrm{~g}, 0.2 \mathrm{~g}$ and $0.25 \mathrm{~g} \mathrm{AN})$. The data reveal that the added of AN to aluminum oxide increases the current. Moreover, the dependence of current on applied volt is linear. Tables 3, 4, 5 and 6 show the liner fitted equations which represent the relation between induced current and applied volt for specific gamma radiation dose. 


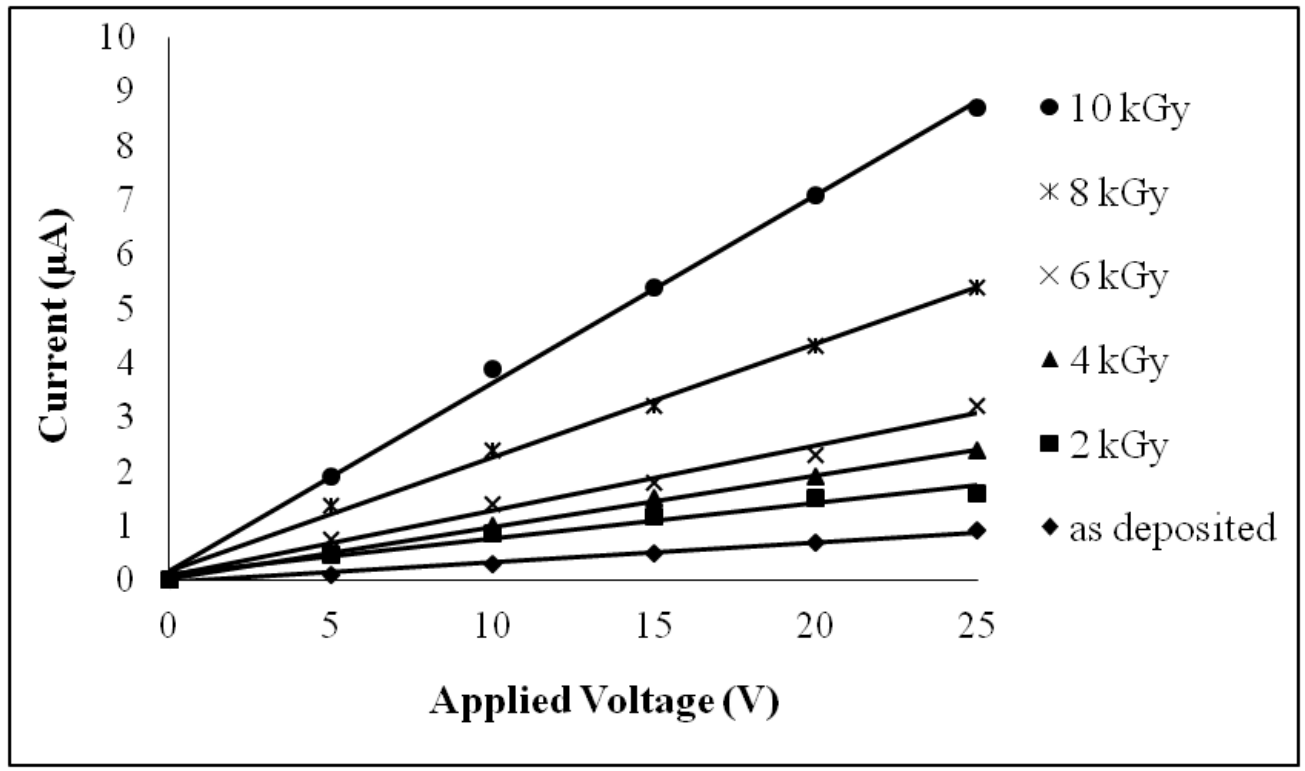

Fig. 5. Typical current-voltage characteristics for Aluminium oxide film doped with 0.1gAN exposed to different levels of the gamma radiation dose.

Table 3. The fitted linear equations represent the relation between induced current and applied volt for Aluminium oxide film doped with $0.1 \mathrm{~g}$ AN

\begin{tabular}{ccc}
\hline $\begin{array}{c}\text { Dose } \\
\mathrm{kGy}\end{array}$ & $\begin{array}{c}\text { Fitted linear } \\
\text { equations }\end{array}$ & $\begin{array}{c}\text { Correlation factor } \\
\left(\mathrm{R}^{2}\right)\end{array}$ \\
\hline 0 & $I=0.037 V-0.0476$ & 0.992 \\
2 & $I=0.064 V+0.1224$ & 0.973 \\
4 & $I=0.095 V+0.0329$ & 0.998 \\
6 & $I=0.120 V+0.0714$ & 0.996 \\
8 & $I=0.209 V+0.1648$ & 0.995 \\
10 & $I=0.3463 V+0.1714$ & 0.997 \\
\hline & $I$ is the induced current $(\mu \mathrm{A})$ and $V$ is applied volt $(V)$
\end{tabular}

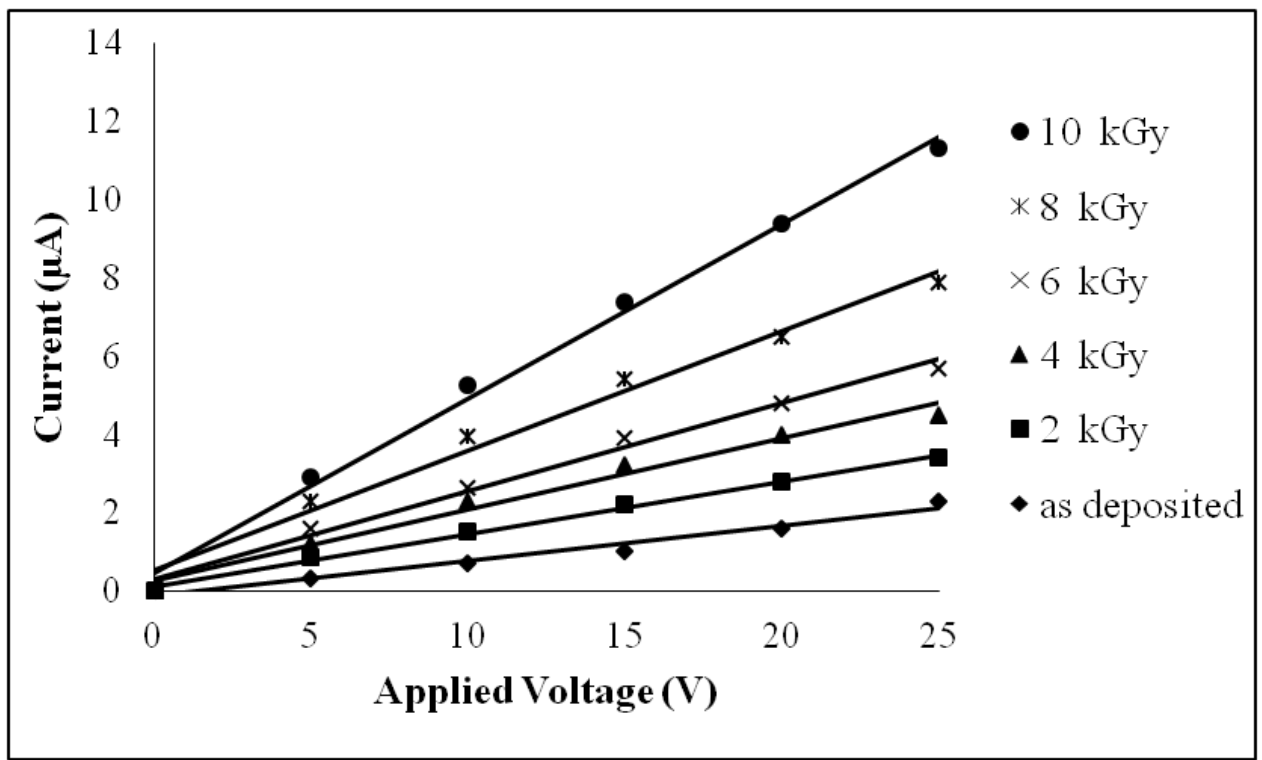

Fig. 6. Typical current-voltage characteristics for Aluminium oxide film doped with $0.15 \mathrm{~g}$ exposed to different levels of the gamma radiation dose 
Table 4. The fitted linear equations represent the relation between induced current and applied volt for Aluminium oxide film doped with $0.15 \mathrm{~g} \mathrm{AN}$

\begin{tabular}{ccc}
\hline $\begin{array}{c}\text { Dose } \\
\mathrm{kGy}\end{array}$ & $\begin{array}{c}\text { Fitted linear } \\
\text { equations }\end{array}$ & $\begin{array}{c}\text { Correlation factor } \\
\left(\mathrm{R}^{2}\right)\end{array}$ \\
\hline 0 & $I=0.0897 V-0.1381$ & 0.971 \\
2 & $I=0.1346 V+0.1095$ & 0.996 \\
4 & $I=0.1817 V+0.2619$ & 0.982 \\
6 & $I=0.2249 V+0.2976$ & 0.989 \\
8 & $I=0.3060 V+0.5167$ & 0.985 \\
10 & $I=0.4466 V+0.4595$ & 0.994 \\
\hline \multicolumn{3}{c}{$I$ is the induced current $(\mu \mathrm{A})$ and $V$ is applied volt $(V)$}
\end{tabular}

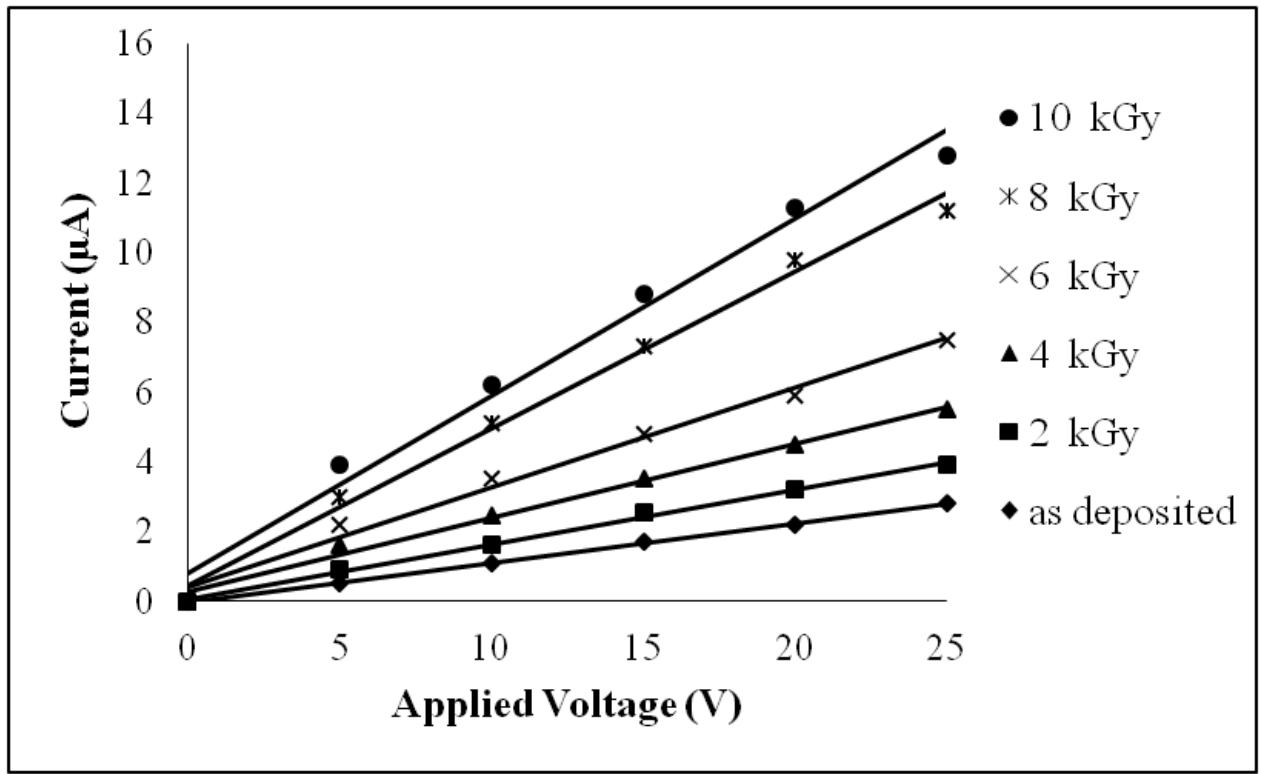

Fig. 7. Typical current-voltage characteristics for Aluminium oxide film doped with $0.2 \mathrm{~g}$ exposed to different levels of the gamma radiation dose

Table 5. The fitted linear equations represent the relation between induced current and applied volt for Aluminium oxide film doped with $0.2 \mathrm{~g} \mathrm{AN}$

\begin{tabular}{ccc}
\hline $\begin{array}{c}\text { Dose } \\
\mathrm{kGy}\end{array}$ & $\begin{array}{c}\text { Fitted linear } \\
\text { equations }\end{array}$ & $\begin{array}{c}\text { Correlation factor } \\
\left(\mathrm{R}^{2}\right)\end{array}$ \\
\hline 0 & $I=0.1126 V-0.0238$ & 0.999 \\
2 & $I=0.1563 V+0.0714$ & 0.996 \\
4 & $I=0.2129 V+0.2643$ & 0.992 \\
6 & $I=0.2851 V+0.4190$ & 0.988 \\
8 & $I=0.4389 V+0.4810$ & 0.994 \\
10 & $I=0.5074 V+0.8238$ & 0.984 \\
\hline \multicolumn{3}{c}{$I$ is the induced current $(\mu \mathrm{A})$ and $V$ is applied volt $(V)$}
\end{tabular}




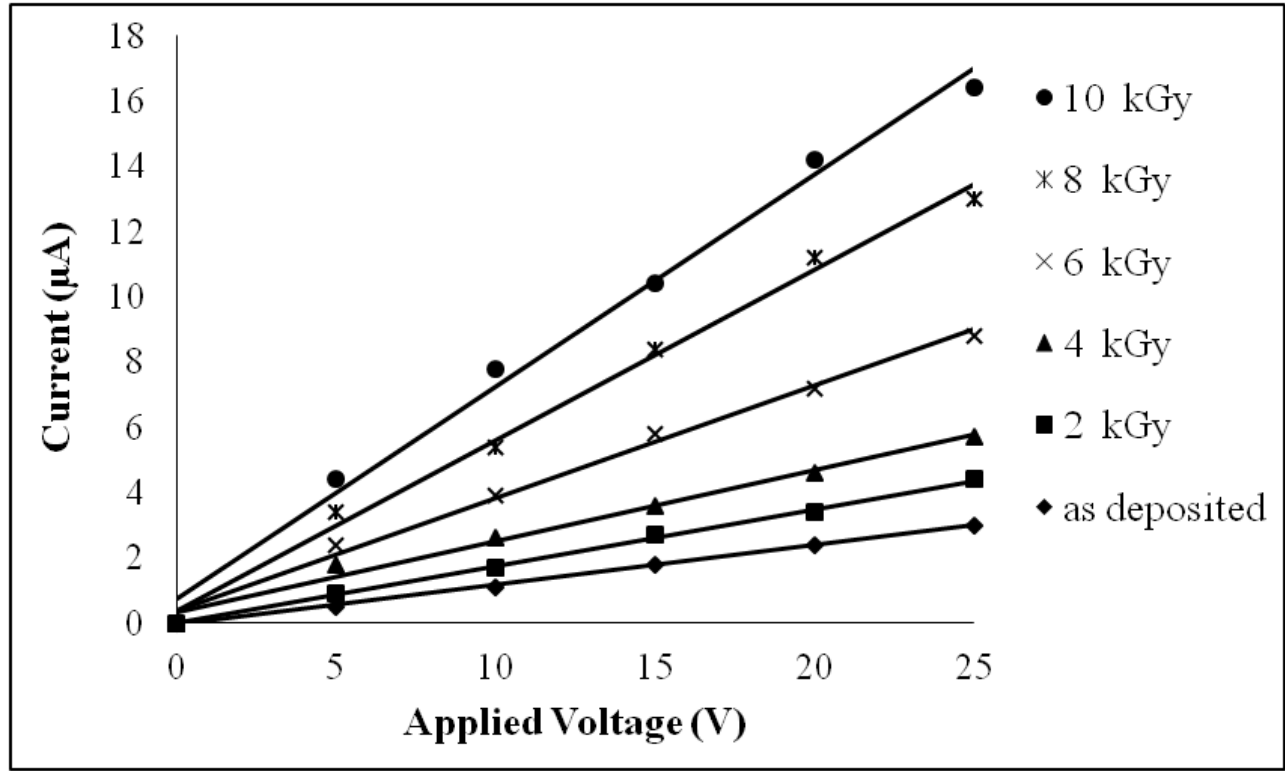

Fig. 8. Typical current-voltage characteristics for Aluminium oxide film doped with $(0.25 \mathrm{~g}$ AN) exposed to different levels of the gamma radiation dose.

Table 6. The fitted linear equations represent the relation between induced current and applied volt for Aluminium oxide film doped with $0.25 \mathrm{~g}$ AN

\begin{tabular}{ccc}
\hline $\begin{array}{c}\text { Dose } \\
\mathrm{kGy}\end{array}$ & $\begin{array}{c}\text { Fitted linear } \\
\text { equations }\end{array}$ & $\begin{array}{c}\text { Correlation factor } \\
\left(\mathrm{R}^{2}\right)\end{array}$ \\
\hline 0 & $I=0.1223 V-0.0619$ & 0.998 \\
2 & $I=0.1743 V+0.0048$ & 0.998 \\
4 & $I=0.2166 V+0.3429$ & 0.986 \\
6 & $I=0.3446 V+0.3762$ & 0.993 \\
8 & $I=0.5223 V+0.3714$ & 0.994 \\
10 & $I=0.6514 V+0.7238$ & 0.992 \\
\hline \multicolumn{3}{c}{$I$ is the induced current $(\mu \mathrm{A})$ and $V$ is applied volt $(V)$}
\end{tabular}

Variations of the current density with the gamma radiation dose at different voltages applied to the coplanar structure of the thin films was obtained as shown in figures 9, 10, 11 and 12 . It is observed that the current density increases, linearly with the gamma radiation dose up to $6 \mathrm{kGy}$ which could be consider as a critical radiation dose. 


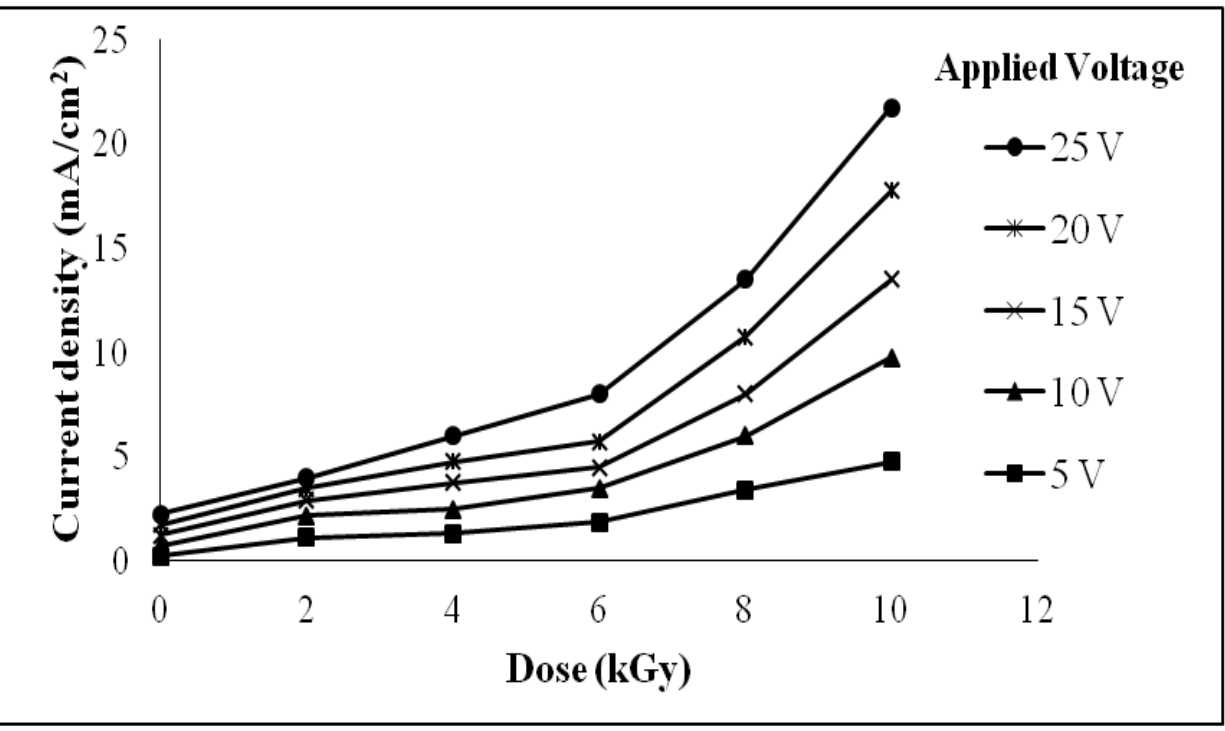

Fig. 9. Variation of the current density with gamma radiation dose for aluminium oxide film doped with $0.1 \mathrm{~g} \mathrm{AN}$

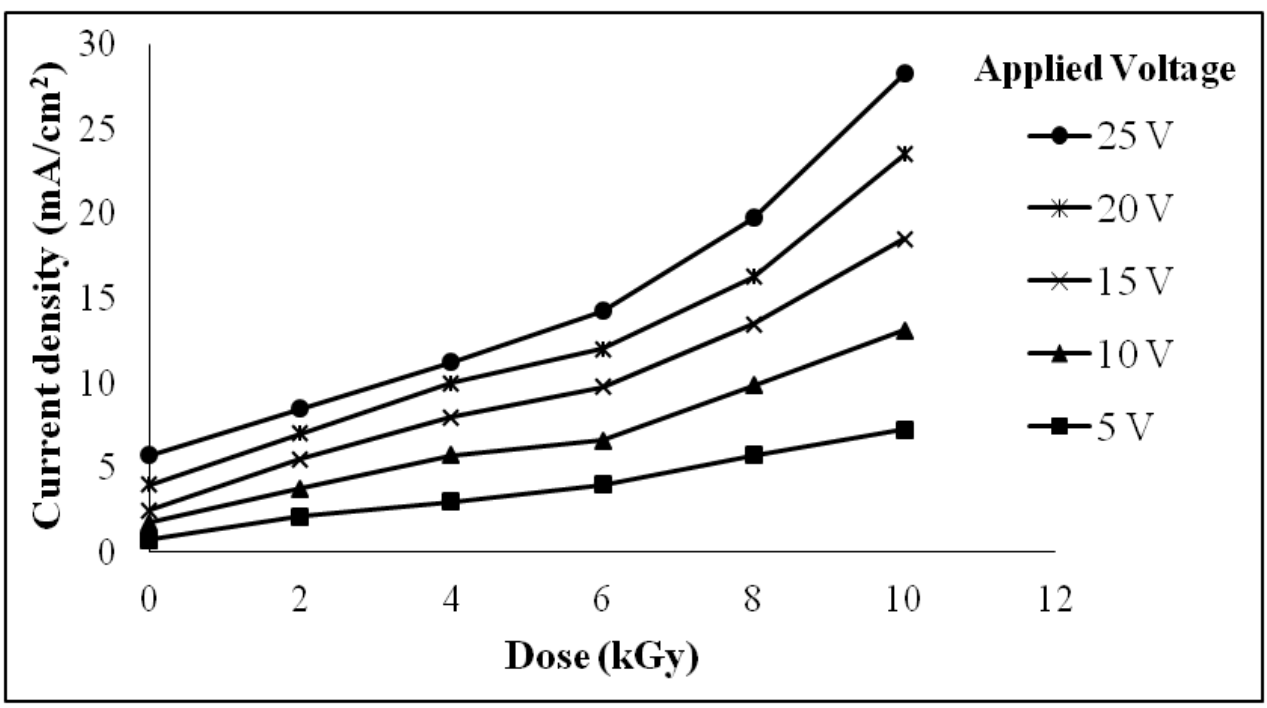

Fig. 10. Variation of the current density with gamma radiation dose for aluminium oxide film doped with $0.15 \mathrm{~g}$ AN 


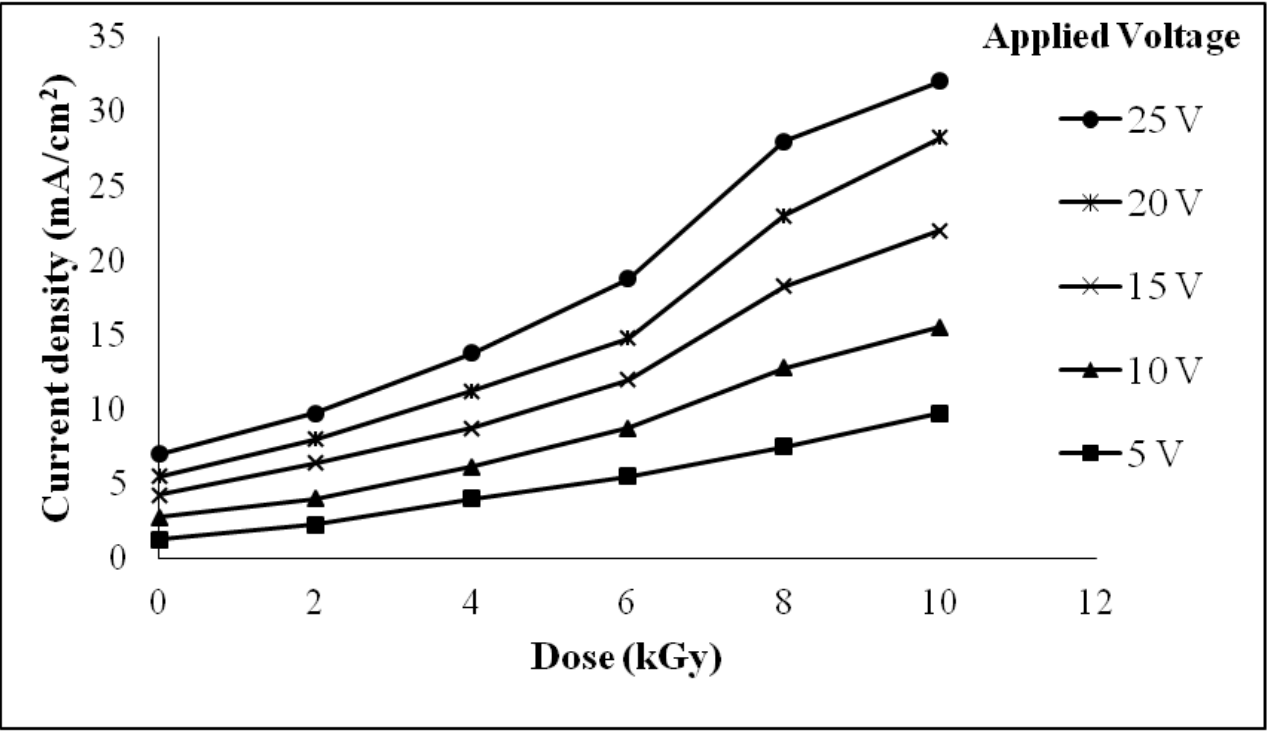

Fig. 11. Variation of the current density with gamma radiation dose for aluminium oxide film doped with $0.2 \mathrm{~g}$ AN

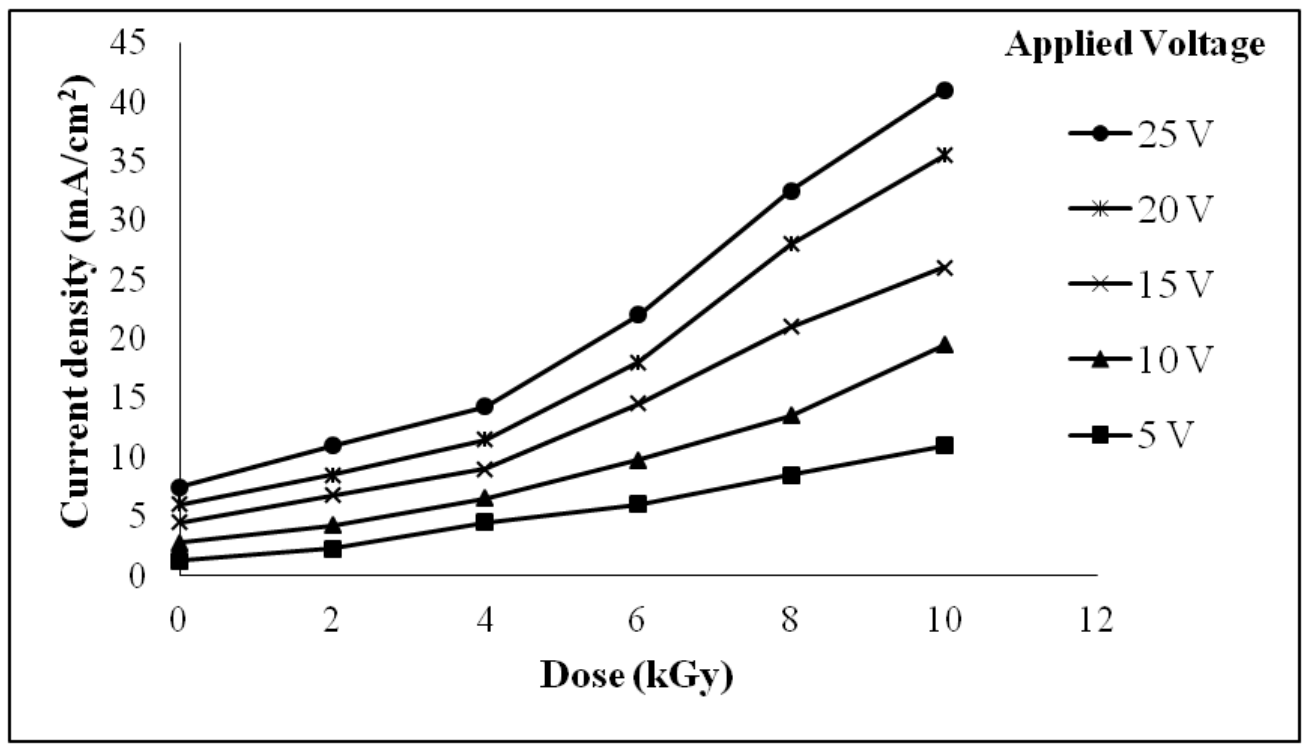

Fig. 12. Variation of the current density with gamma radiation dose for aluminium oxide film doped with $0.25 \mathrm{~g}$ AN

Sensitivity representing the change in the current density per unit change of the gamma radiation dose was estimated over the linear regions. Correspondingly, the detection limit was defined as the gamma radiation dose required for producing a unit change in the current through a film of a particular cross sectional area. From that study, the sensitivity of the detector film versus the applied voltage increase as the AN content increase in the detector films as shown in figure 13. 


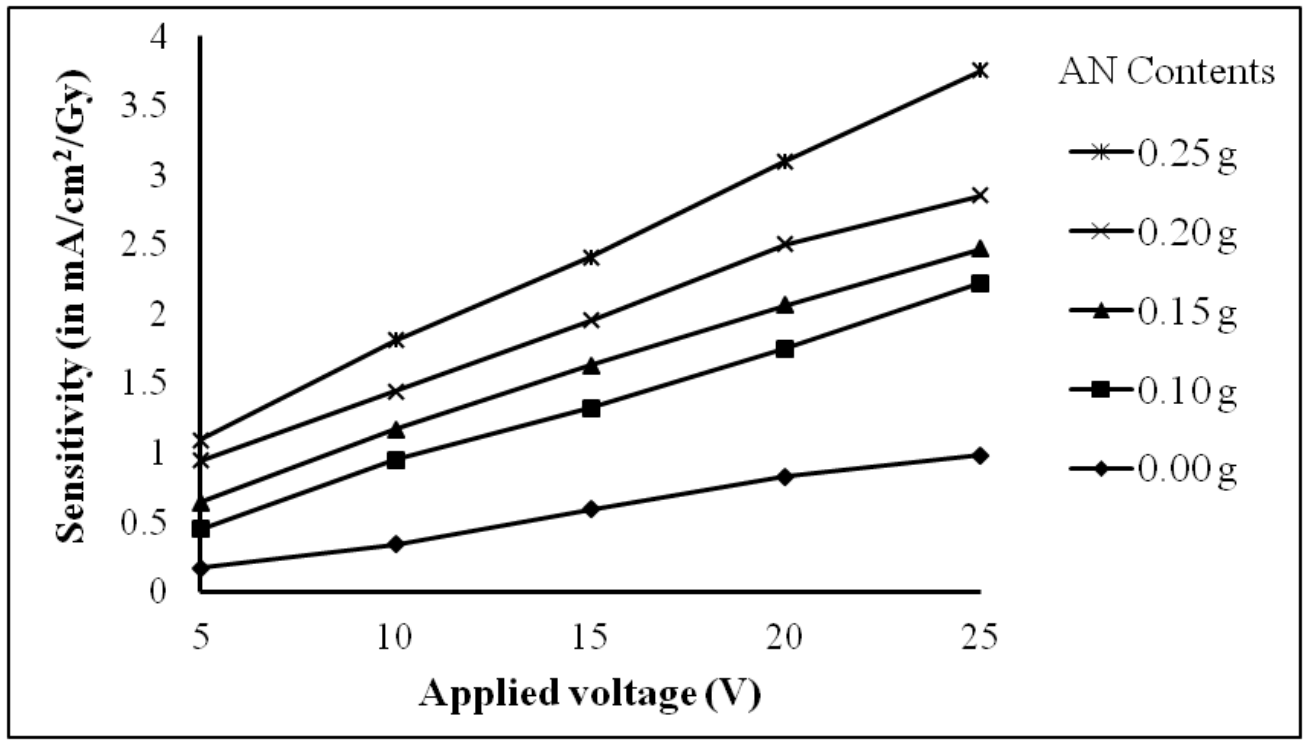

Fig. 13. Sensitivity for gamma radiation for different films of different AN content

Effect of the AN can be accounted for polymerization of polyaniline, which affect the conductivity of the films and variation with gamma radiation is shown in figure 14 .

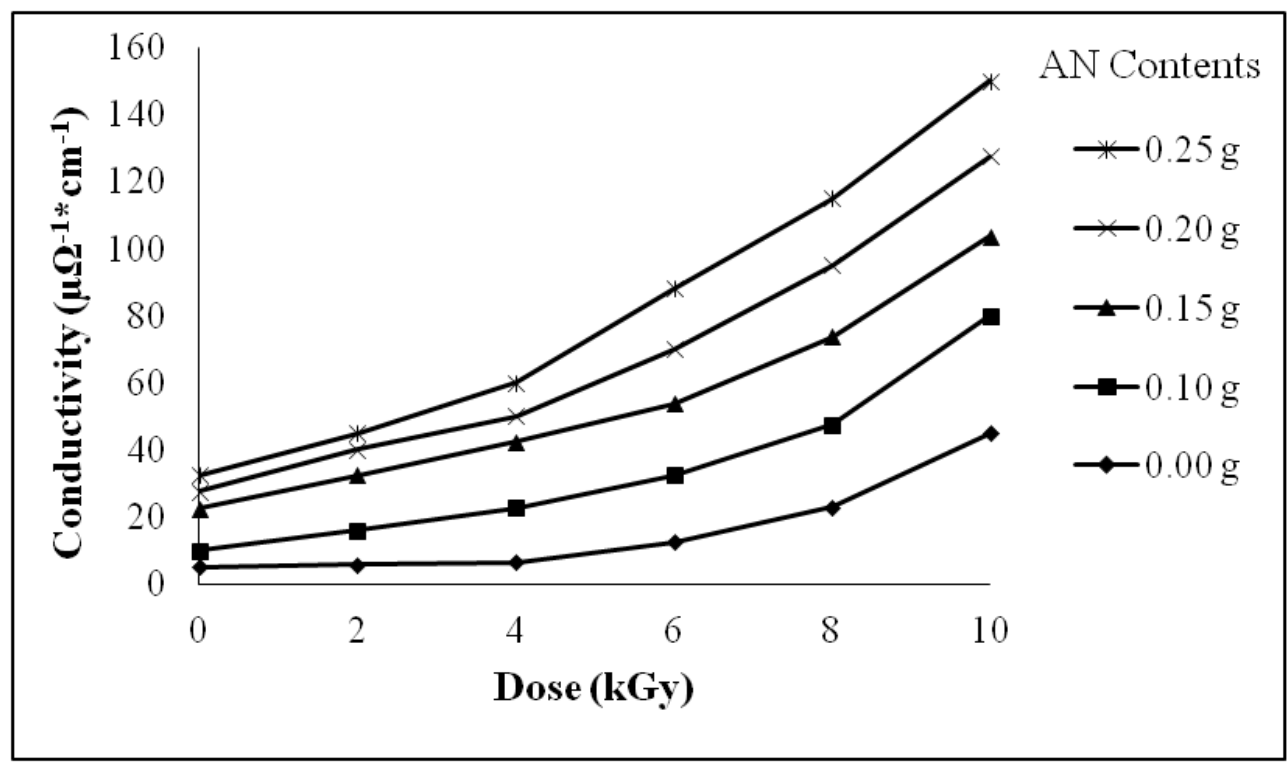

Fig. 14. Conductivity for different films at different AN content due to gamma radiation

\section{Conclusion}

Metal oxide films are very promising field of research for gamma radiation dosimetery. In this work, the simple sol-gel technique was used for the preparation of aniline doped aluminum oxide films for the purpose of gamma radiation dosimetry AN was suggested to enhance films conductivity and sensitivity to radiation. Films conductivity and sensitivity to gamma radiation was studied in the $0-10 \mathrm{kGy}$ dose range with AN content of the range $0-0.25 \mathrm{~g}$ with varying voltage bias in the range of $0-25 \mathrm{~V}$. Electrical conductivity and sensitivity was found to increase linearly with polyaniline doping concentration and dose, high conductivity and sensitivity values up to $150 \mu \Omega^{-1} \cdot \mathrm{Cm}^{-1}$ and $3.75\left(\mathrm{~mA} / \mathrm{cm}^{2} / \mathrm{kGy}\right)$ respectively were achieved at $0.25 \mathrm{AN}$ content and $25 \mathrm{~V}$ bias. 


\section{References}

[1] Khalil Arshak et al. "Response of metal oxide thin film structures to radiation". Materials Science and Engineering B 133 (2006).

[2] J. Corcoran et al. "Gamma radiation sensing properties of $\mathrm{TiO}_{2}, \mathrm{ZnO}, \mathrm{CuO}$ and $\mathrm{CdO}$ thick film pn-junctions". Sensors and Actuators A 123-124 194-198 (2005).

[3] K. Arshak, O. Korostynska, E. Jafer, A. Arshak, D. Morris and E. Gill. "Gamma Radiation Sensing Using $\mathrm{ZnO}$ and $\mathrm{SnO} 2$ Thick Film Interdigitated Capacitors". IEEE, 2006.

[4] K. Arshak, O. Korostynska and Farah Fahim. "Various structures based on nickel oxide thick films as gamma radiation sensor". Sensors 3, 176-186, 2003.

[5] K. Arshak, O. Korostynska. "Thick film oxide diode structures for personal dosimetry application". Sensors and Actuators A 113 319-323 (2004).

[6] K. Arshak, O. Korostynska. "Preliminary studies of properties of oxide thin/thick films for gamma radiation dosimetry". Materials Science and Engineering B107 224232 (2004).

[7] T.K. Maity, S.L. Sharma, G. Chourasiya. "The real-time gamma radiation dosimetry with TeO2 thin films". Radiation Measurements 47 145-148 (2012).

[8] Ercan Yilmaz. "Use of $\mathrm{Al}_{2} \mathrm{O}_{3}$ layer as a dielectric in MOS based radiation sensors fabricated on a Si substrate". Nuclear Instruments and Methods in Physics Research B $2664896-4898$ (2008).

[9] Ziran Liu, Wenbin Guo, Daguang Fu, Weiyou Chen. "p-n Heterojunction diodes made

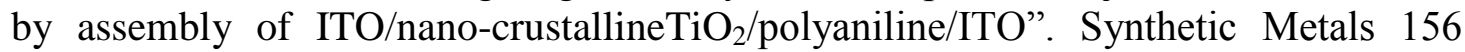
414-416 (2006).

[10] Olgun Guven. "Radiation-induced conductivity control in polyaniline blends/composites". Radiation Physics and Chemistry 76 1302-1307 (2007).

[11] Neal Alfie Y. Lasta, and Editha P. Jacosalem. "Polyaniline pellets: radiation sensor". $8^{\text {th }}$ SPVM National Physics Conference 2007.

[12] Ana P. Lima Pachecoa, Elmo S. Araujoa, Walter M. de Azevedo. "Polyaniline/poly acid acrylic thin film composites: a new gamma radiation detector". Materials Characterization 50, 245-248, (2003).

[13] R G Sonkawade. "Effect of gamma ray and neutron radiation on polyaniline conducting polymer". Indian journal of pure \& Applied Physics. Vol. 48, pp. 453-456, July 2010.

[14] A. Omar, A. Baraka, A. H. Zaki, and K. A. Sharshar, "Gamma Radiation Effect on the Electrical Properties of PANI Film". Applied Mechanics and Materials, vol. 241, pp. 828-832, 2013. 